\title{
PERFORMANCE ANALYSIS OF EDFA BASED ON GAIN WITH DIFFERENT PARAMETERS
}

\author{
Himanshu Singh ${ }^{1}$, S.Sugumaran ${ }^{2}$ \\ ${ }^{I}$ Student, Sece, Galgotia University, U.P, India. \\ ${ }^{2}$ Assistent Professor, Sece, Galgotias University, U.P, India.
}

\begin{abstract}
The optical fiber amplifier in active medium consist of 10-30(m) length of fiber which is lightly doped with the rare earth element, for example, ytterbium(yb) Erbium(Er). For the long distance optical communication, EDFA is used effectively, the material which is made by doping silica with erbium. For rising pumping efficiency and amplifier gain Yb is added. Standard EDFA normally operated in the 1530-1565nm region. In this paper the variation of fiber length w.r.t various parameter has been analysis and based on gain and noise figure value is analysis with MATLAB software.
\end{abstract}

Keywords: Gain, EDFA, Optical Fiber, Noise Figuere.

\section{INTRODUCTION}

In the recent year, due to dramatic reduction in the transmission bandwidth property we use optical fiber. optical fiber are use because of some property of optical fiber like data security, small size, high bandwidth, ease of installation, immune to electromagnetic effect.

EDFA are the most part utilize as preamplifier with multichannel amplification without crosstalk and also multi -gigabit transmission rates by low bit error. Most significant technology is the (EDF), which traditionally silica doped fiber along erbium .since EDFA work in near wavelength of 1.55 micro-meter it become famous . Deployment of EDFA led to light wave system capacity exceeding 1TB/S.

The execution characteristic of EDFA running in c- band and pumped at $980 \mathrm{~nm}$ recreated. Gain and noise figure change were gotten as action of various parameter [1]. A two phase combination fiber amplifier structure was utilized with $1555 \mathrm{~nm}$ high power fiber amplifier with various length of increase fiber [2]. In this we analyze gain against input power for EDFA, To calculate the input pump power and gain for EDFA, R-K method is used, And compare the theoretical result with experimental characterization for gain versus pump power. The systematic approach is used to determine EDFA gain for the action of input pump power[3]. In that we analyze fiber transmission penalties cost EDFA gain transient in a chain of 10-20 EDFA with as many as 32 wavelength-divisionmultiplexed channels. Penalties are a result of increase fiber non-linearity during channel deletion and amplified spontaneous emission noise during channel addition [4]. This paper speak to all available triple pass EDFA structure execution of all the setup has been investigated with the output power, input power, maximum input power, for whole shape from the execution, best triple pass and also multistage EDFA has been outline to produce optimum gain small noise figure[5]. EDFA arrange in double stage scheme in which triple band pass filter is added, to reflect the amplified signal back to EDF circulator is used.TBF which filter out ASE which provide profitable amplification of the signal which travel along the fiber[6]. In this study we evaluate fundamental impact of EDFA with large gain and low noise figure[7].

\section{PERFORMANCE ANALYSIS}

In the performance analyses increase the gain and reduce the noise figure. As the gain of the system was $35 \mathrm{db}$ and noise figure was $6.1 \mathrm{db}$ initially, but we have increase the gain up to $35.35 \mathrm{db}$ and noise figure become reduce to $4.41 \mathrm{db}$. The performance is done with the help of Runga Kutta method of order fourth. The differential equation we utilize RUNGA-KUTTA strategy for request fourth, since it gives correct result we utilize R-K method. The variation of fiber length with respect to various parameters has been analyzed, all the analysis has been done with the MATLAB Software. In figure 1 , to study the change of gain against fiber, the length of the fiber is $14 \mathrm{~m}$, from the graph it has been analyzed that as the fiber length increase the gain is increased linearly after that as the fiber length increase the gain of the system decrease, the region for low value of gain is due to unsatisfactory population inversion then present gain at signal wavelength as a result of high total loss of erbium doped fiber. In the fig (2), the change of fiber length along signal ASE has been plotted, the signal ASE works in both forward ASE and backward ASE, in the forward ASE, as the fiber length increased the forward AES decrease exponentially as the fiber length increased, and in the case of backward ASE as the fiber length increased the backward ASE increased exponentially but after further increase the fiber length the backward ASE decrease. Both the forward ASE and backward ASE are reverse to each other, forward ASE and backward ASE both are taken together because ASE effect reduction in amplifier gain and increment in noise figure. In fig(3), it show the change of fiber length against output power, as the fiber length increase the 
signal power increase but as the fiber length increase further the output power decrease, as the output power is increase gain is also increase because gain is equal to output power upon input power, so if the output power is increase gain is also increase and fiber length is also increase. In fig (4), the input power immediately attenuate along fiber length the region is, erbium consumption and traditional loss of silica. In fig(5), it shows the change of gain vs. input power, as the gain rises input power also increment after a specific point increment in gain is low, the region is when the population inversion is given for all the erbium ion in the fiber, if we want excellent gain then we have to use lengthy erbium doped fiber with effective pumping. In fig (6), show the variation of pump power with respect to signal power, it show that as the pump power increase signal power increase but as the value of the pump power increase signal power decrease. There is no specific time the change of input power accordingly with the output power , the variation of pump power accordingly with signal power.

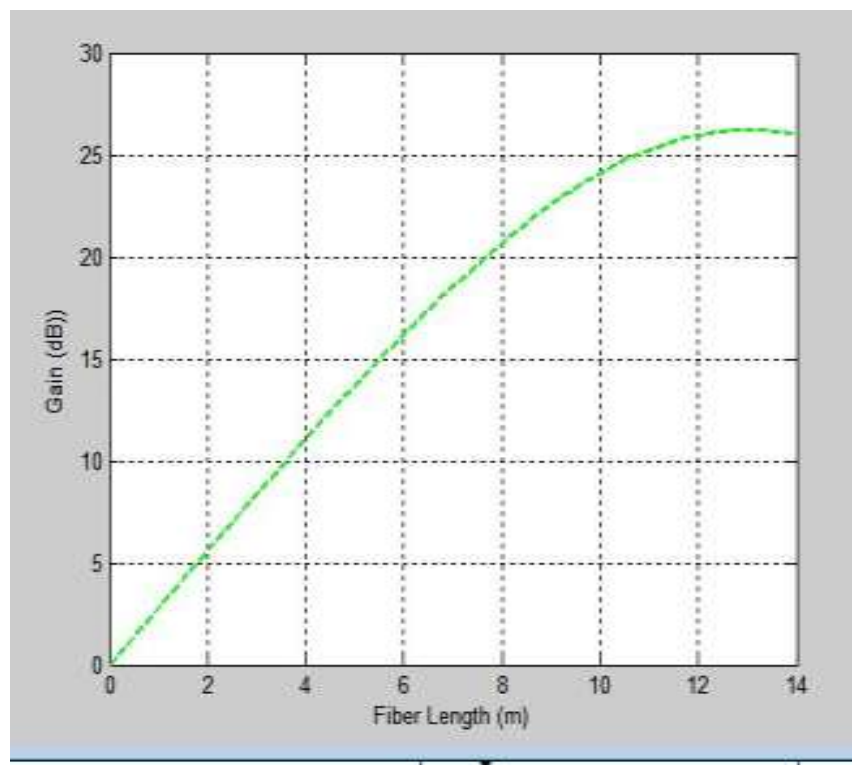

Fig(1)-show the variation between fiber length and gain

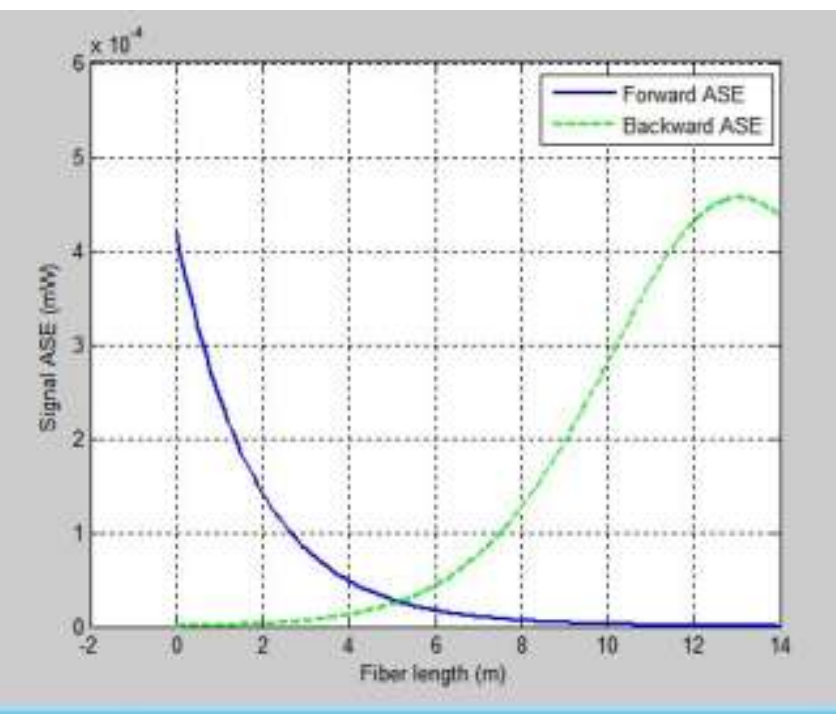

Fig(2)- show the variation between fiber length and signal ASE.

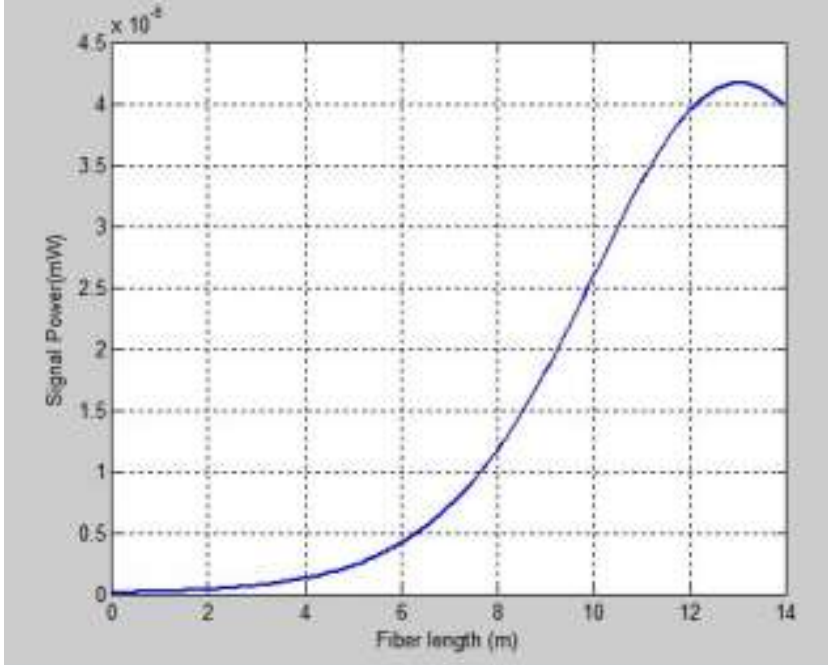

Fig(3)-show the variation between fiber length and signal power.

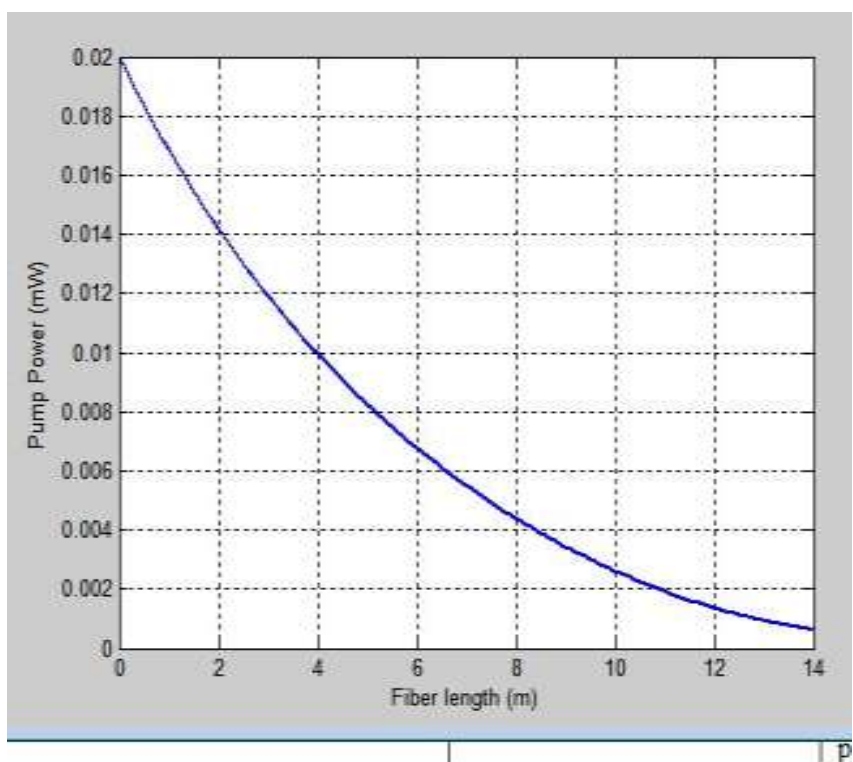

Fig(4)-show the variation between fiber length and pump power.

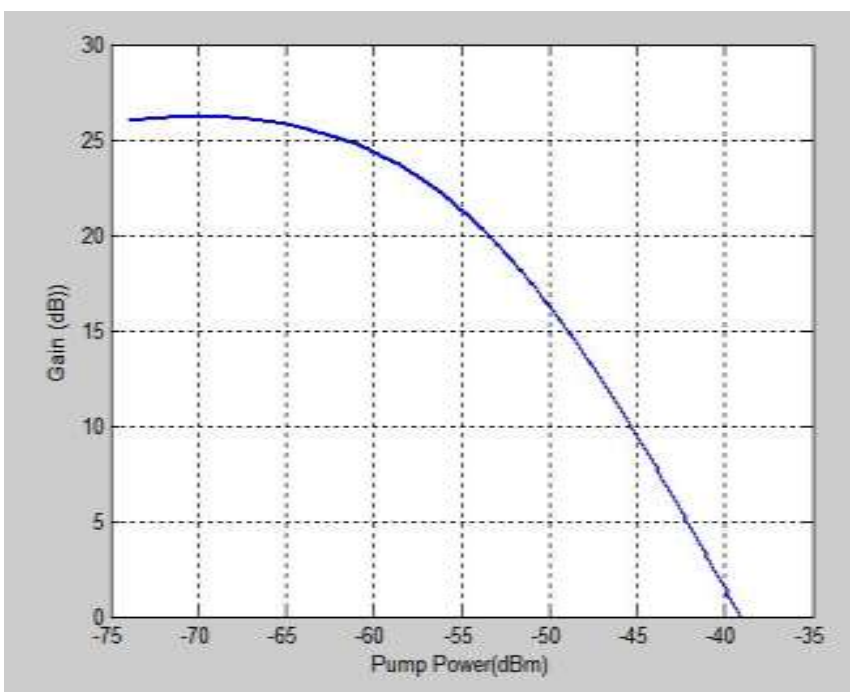

Fig(5)-show the variation between pump power and gain. 


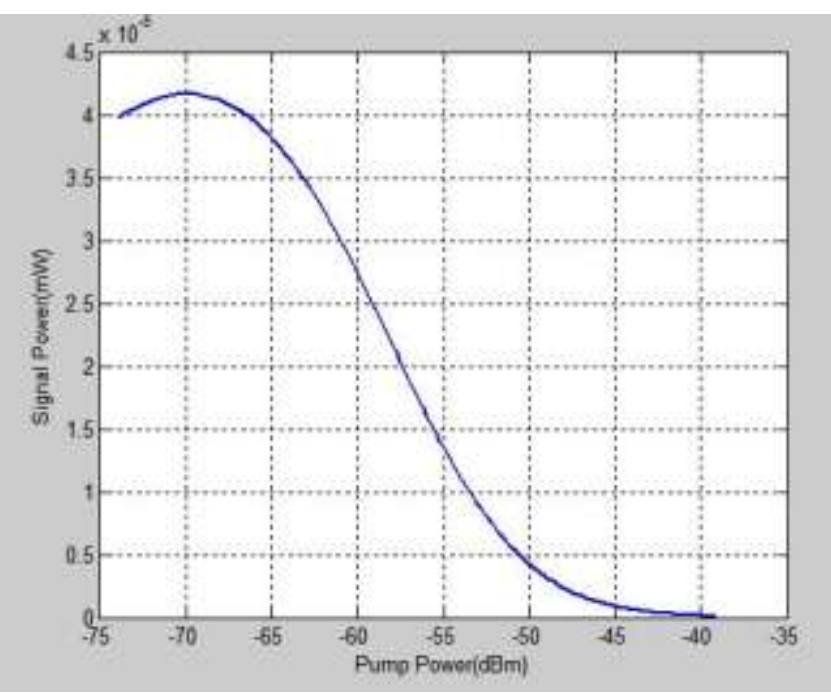

Fig (6)-show the variation between pump power and signal power.

\section{3-METHODOLOGY USED}

To determine the differential equation we utilize RUNGAKUTTA strategy for request fourth, since it gives correct result we utilize RUNGA-KUTTA method

$\mathrm{M}_{1}=\mathrm{s} \mathrm{p}\left(\mathrm{r}_{0}, \mathrm{k}_{0}\right)$;

$\mathrm{M}_{2}=\mathrm{s} \mathrm{p}\left(\mathrm{r}_{0}+1 / 2 \mathrm{~s}, \mathrm{k}_{0}+1 / 2 \mathrm{~m}_{1}\right)$;

$\mathrm{M}_{3}=\mathrm{s} \mathrm{p}\left(\mathrm{r}_{0}+1 / 2 \mathrm{~s}, \mathrm{k}_{0}+1 / 2 \mathrm{~m}_{2}\right)$;

$\mathrm{M}_{4}=\mathrm{s} \mathrm{p}\left(\mathrm{r}_{0}+\mathrm{h}, \mathrm{k}_{0}+\mathrm{m}_{3}\right)$;

And finally we calculate the value of $\mathrm{m}$,

$M=1 / 6\left(m_{1}+2 m_{2}+2 m_{3}+m_{4}\right)$.

\section{APPLICATION}

The application of Erbium doped fiber amplifier (EDFA) are important technology for the video, data transmission, light wave voice. As the passage of 1996 telecommunication act and growth of the internet has been demand for the extended bandwidth in all network layer resulting in advance in Erbium doped fiber technology.

\section{CONCLUSION}

In this paper, we described the performance analysis of EDFA.Any system which has high gain is its advantage. As we know that gain is inversely proportional to Noise figure, so as the noise figure will decrease so gain will improve. The gain of the system was $35 \mathrm{~dB}$ initially then the Noise figure was $6.1 \mathrm{~dB}$ approx but when we have increased the gain up to $35.35 \mathrm{~dB}$ then the noise figure suddenly decreased up to $4.41 \mathrm{~dB}$. And performance analysis of EDFA for various parameters has been done.

\section{REFERENCES}

[1]. Dhokar, Deshmukh 'Overview Of Edfa For The Efficient Performance Analysis' Journal of Engineering, Vol.04, Issue03 (March 2014)

[2]. Wang Ji, Li Nan 'High Power ER3+/YB3+ Co-Doped Fiber Amplifier Of Fiber Length Optimization'(IEEE 2010).
[3]. Paul Schiou, Floren Vasile 'The Edfa Performance With Gain Versus Pump Power',(IEEE 2004).

[4]. M.I.Hayee And A.E. Willner 'Transmisition Penalties Due to EDFA Gain In Add- Drop Multiplexed WDM Network'(IEEE 1999).

[5]. Fowzia Akhter ,A.W.Naji, M.I .Ibrahimy And H.R. Siddiquei, 'Characterization of Triple Pass EDFA',(ICCCE 2012).

[6]. Khalida.S.Al- Khateeb M.A. Mohammad, A.W.Naji H.F.H .Ibrahim 'Theoretical Analysis Of A Double Stage EDFA'.(ICCCE 2010).

[7]. Rahul Singh Chauhan, S. Sugumaran 'Basic Analysis Of Gain In Thermo of Noise Figure', IJAEGT,vol-03 (May 2015). 